\title{
The Equivalence of Inverse Compton Scattering and the Undulator Concept
}

\author{
K.Y. Ng \\ Fermi National Accelerator Laboratory, Batavia, IL 60510
}

August, 2009

\begin{abstract}
Inverse Compton scattering is a method to produce very high frequency photon beam. However, the production mechanism can also be viewed as a undulator emission. This is because the electron sees electric and magnetic fields of the incident laser beam and is driven into transverse oscillatory motion in exactly the same way when the electron passes through a undulator consisting of alternating magnetic field. This note gives a detailed examination of the similarity about the two views. Equivalent undulator parameters are derived for the incident laser beam, as well as the differential cross section of photon emission.
\end{abstract}




\section{Introduction}

Undulator emission is a mechanism to generate short-wavelength photon beam. An electron going through a undulator of period or wavelength $\lambda_{u}$ emits radiation with wavelength $\lambda_{s} \approx$ $\left(1+K^{2} / 2\right) \lambda_{u} /\left(2 \gamma^{2}\right)$ in the forward direction, where $K_{u}$ is the undulator parameter and $\gamma$ is the relativistic factor of the electron. The reduction in wavelength by the factor $2 \gamma^{2}$ is tremendous. Unfortunately, due to technical difficulties, the undulator period cannot be manufactured to much less than the order of a centimeter, which therefore limits the frequency of the photon generated at a fixed electron energy.

An electron colliding with a laser beam sees alternating electric and magnetic fields of period $\sim \lambda_{L} / 2$, where $\lambda_{L}$ is the wavelength of the laser beam. Thus the electron will be driven into oscillatory transverse motion in exactly the same way as inside a undulator. The equivalent undulator period $\sim \lambda_{L} / 2$ is obvious very much smaller than the period of the mechanical undulator. As a result, the photons emitted in the forward direction will have the much smaller wavelength of $\sim \lambda_{L} /\left(4 \gamma^{2}\right)$.

The above description can also be considered as a laser beam colliding with a moving electron head-on and reversing its direction after scattered by the electron. This process is called inverse Compton scattering. Thus inverse Compton scattering can also be described by the language of undulator emission. In this note, we give a detailed examination of the similarity about the two views. The limitation of the similarity of the two views are also sighted. Equivalent undulator parameters are derived for the incident laser beam, as well as the differential cross section of photon emission.

\section{Inverse Compton Scattering}

The electron-storage ring at IUCF of Indiana University will supply an electron beam with $\gamma=100$. A laser beam with optical wavelength $\lambda_{L}=10000 \AA$ collides with the electron bunches in the opposite direction. We have laser wavelength $\lambda_{L}=10^{-6} \mathrm{~m}$ and frequency $f_{L}=0.3 \times 10^{9} / 10^{-6}=3 \times 10^{14} \mathrm{~Hz}$. Now transform to the rest frame of the electron. The laser photon will increase in energy, which becomes

$$
f_{L 0}=\gamma f_{L}(1+\beta) \approx 2 \gamma f_{L}=6 \times 10^{16} \mathrm{~Hz}
$$


and wavelength

$$
\lambda_{L 0}=\frac{c}{f_{L 0}} \approx \frac{c}{2 \gamma f_{L}}=\frac{\lambda_{L}}{2 \gamma}=5 \times 10^{-9} \mathrm{~m}=50 \AA .
$$

This is the frame (electron initially rest) Compton scattering is usually studied in the old days. In this frame, the scattered photon has the energy

$$
h f_{s 0}=\frac{h f_{L 0}}{1+\frac{h f_{L 0}}{m_{e} c^{2}}\left(1-\cos \theta_{e}\right)} .
$$

Here, $\theta_{e}$ is the scattered angle of the scattered photon in the rest frame of the electron. It is defined in such a way that a forward scattered photon (no direction change) has $\theta_{e}=0$, while a backward scattered photon (direction reversed) has $\theta_{e}=\pi$. Obviously, the scattered photon that reverses its incident direction has the lowest energy, which equals

$$
h f_{s 0}=\frac{h f_{L 0}}{1+\frac{2 h_{0} f_{L 0}}{m_{e} c^{2}}} \approx h f_{L 0},
$$

since for a $6 \times 10^{16} \mathrm{~Hz}$ photon, $h f_{L 0}=2.48 \times 10^{-4} \mathrm{MeV}$, where $h=4.1357 \times 10^{-21} \mathrm{MeV}-\mathrm{s}$ is the Planck constant. With $h f_{L 0} / m_{e} c^{2} \ll 1$, Compton scattering just reduces to Thomson scattering, and we call this the Thomson limit,

Now going back to the lab frame, this backward scattered photon moves in the direction of the electron with frequency

$$
f_{s}=f_{s 0} \gamma(1+\beta) \approx 2 \gamma f_{s 0} \approx 2 \gamma f_{0} \approx 4 \gamma^{2} f_{L}
$$

$\mathrm{Or}$

$$
\frac{f_{L}}{f_{s}}=\frac{1}{(1+\beta)^{2} \gamma^{2}}
$$

The scattered photon frequency is $f_{s} \approx 4 \gamma^{2} f_{L}=1.2 \times 10^{19} \mathrm{~Hz}$ and the wavelength is $\lambda_{s}=\lambda_{L} / 4 \gamma^{2}=2.5 \times 10^{-11} \mathrm{~m}=0.25 \AA$. Notice that the change has been by the factor $4 \gamma^{2}$, which is very big. Compton scattering in the moving frame of the electron with the photon beam reversing direction is called inverse Compton scattering.

For a scattered photon in the general direction $\theta_{e}$, its scattered direction $\theta_{\ell}$ in the lab frame is given by (see Appendix)

$$
\cos \theta_{e}=\frac{\gamma^{2} \beta \tan ^{2} \theta_{\ell}-\sec \theta_{\ell}}{1+\gamma^{2} \tan ^{2} \theta_{\ell}}
$$

where the subscript $e$ designates the frame of reference where the electron is at rest, while the subscript $\ell$ designates the lab frame where the undulator is at rest. Here, $\theta_{\ell}$ is defined 
in the forward direction of the electron; i.e., $\theta_{\ell}=0$ implies the scattered photon is emitted exactly in the forward direction of the electron or the reverse direction of the laser beam. For large $\gamma, \theta_{\ell}$ is small, and the above can be approximated by

$$
\cos \theta_{e} \approx-\left\{1-\left[\gamma^{2}(1+\beta)-\frac{1}{2}\right] \theta_{\ell}^{2}\right\} \approx-\left(1-2 \gamma^{2} \theta_{\ell}^{2}\right) .
$$

Since the photon energy is transformed to the lab frame by

$$
h f_{s}=\gamma\left(1-\beta \cos \theta_{e}\right) h f_{s 0}
$$

the scattered photon will have frequency $f_{s}$ in the lab frame given by

$$
\frac{f_{s}}{f_{L}} \approx \frac{\gamma^{2}(1+\beta)\left(1-\beta \cos \theta_{e}\right)}{1+\frac{h f_{L}}{m_{e} c^{2}} \gamma(1+\beta)\left(1-\cos \theta_{e}\right)} .
$$

Substituting for $\cos \theta_{e}$, we get

$$
\frac{f_{s}}{f_{L}} \approx \frac{\gamma^{2}(1+\beta)^{2}\left[1-\frac{1}{2} \beta(1+\beta) \gamma^{2} \theta_{\ell}^{2}\right]}{1+\frac{2 h f_{L}}{m_{e} c^{2}} \gamma(1+\beta)\left[1-\left(\frac{1}{2} \gamma^{2}(1+\beta)-\frac{1}{4}\right) \theta_{\ell}^{2}\right]} .
$$

In above, we have only made the small $\theta_{\ell^{-}}$expansion, but have kept all orders of $(1-\beta)$. Since $h f_{L} / m_{e} c^{2} \ll 1$, we can let $\beta \rightarrow 1$ in the denominator. We can then write the wavelengths of the scattered photon to the initial photon as

$$
\frac{\lambda_{s}}{\lambda_{L}} \approx \frac{1+\frac{4 h f_{L}}{m_{e} c^{2}} \gamma\left(1-\gamma^{2} \theta_{\ell}^{2}\right)+\frac{1}{2} \beta(1+\beta) \gamma^{2} \theta_{\ell}^{2}}{\gamma^{2}(1+\beta)^{2}}
$$

Consider an electron bunch of length 1 ps or $0.3 \times 10^{-3} \mathrm{~m}(0.3 \mathrm{~mm})$ containing $N_{b}=10^{12}$ particles. In each wavelength of the scattered laser beam, number of electrons is on the average

$$
N_{\lambda}=\frac{N_{b} \times 2.5 \times 10^{-11}}{0.3 \times 10^{-3}}=8.33 \times 10^{4}
$$

\section{Interaction with Laser Beam}

If the laser beam and electron are moving in the same direction, there should be no interaction. Suppose that the direction of propagation is $z$. At on moment, an electron sees a $E$ field in the $x$-direction and a $B$ field in the $y$-direction. The electron is subject to the electric force $e E$ in the positive $x$-direction, and a magnetic force $v \times B$ which is the negative $x$-direction. Since the electron velocity is close to $c$, the electric and magnetic forces cancel each other. On the other hand, if the laser beam is propagation in the direction opposite to that of the electron, the magnetic and electric forces add. 


\section{Undulator Theory}

Microscopically, we can view Compton scattering as an undulator theory. An electron sees the laser beam in the lab frame as an undulator because the electron sees a magnetic field that is alternating. The electron is set to transverse oscillation as a result of seeing the electric and magnetic components of the laser beam. The synchrotron radiation emitted by the electron is just the scattered photon ray of Compton scattering. However, there are two differences from the conventional undulator theory:

\section{Undulator Period}

The equivalent undulator period $\lambda_{u}$ is not equal to the wavelength $\lambda_{L}$ of the laser beam, because the photons from the laser beam is moving towards the electron. Let $t$ be the equivalent undulator period in time, or $t$ is the time the electron sees another wave crest of the laser beam after passing the previous wave crest. We have

$$
t=\frac{\lambda_{u}}{\beta c}=\frac{\lambda_{L}-\lambda_{u}}{c}
$$

where $\lambda-\lambda_{u}$ is the distance the wave crest of the laser beam moves in time $t$ to meet the electron. Thus

$$
\lambda_{u}=\frac{\beta \lambda_{L}}{1+\beta} \approx \frac{\lambda_{L}}{2}
$$

\section{Undulator magnetic field}

As mentioned above, since the laser photon and the electron are traveling in opposite direction, the electric and magnetic forces enhance each other. For a plane wave, electric field strength $\vec{E}$ and magnetic field strength $\vec{B}$ are related by

$$
\omega \vec{B}=\vec{k} \times \vec{E}
$$

where $\vec{k}$ is the propagation vector having magnitude $|\vec{k}|=\omega / c$. The magnetic field of the equivalent undulator is just

$$
B_{u}=B+\frac{E}{\beta c}=\frac{(1+\beta) B}{\beta} \approx 2 B
$$


Thus at an angle $\theta_{\ell}$ in the lab frame in the direction of the electron, the wavelength of radiation $\lambda_{s}$ is

$$
\frac{\lambda_{s}}{\lambda_{u}}=\frac{k_{u}}{k_{s}}=\frac{1+(1+\beta) K^{2} / 4+\beta(1+\beta) \gamma^{2} \theta_{\ell}^{2} / 2}{\beta(1+\beta) \gamma^{2}}
$$

where $k_{u}=2 \pi / \lambda_{u}$, with the equivalent undulator parameter

$$
K_{u}=\frac{e B_{u}}{m_{e} \beta c k_{u}}=\frac{e B_{u} \lambda_{u}}{2 \pi m_{e} \beta c}=\frac{e B \lambda_{L}}{2 \pi m_{e} \beta c}=\frac{e B}{m_{e} \beta c k}
$$

In Eqs. (4.5) and (4.6), we have tried not to let $\beta \rightarrow 1$. We see that the undulator parameter is exactly the same if we substitute the equivalent magnetic field $B_{u}$ by $B$, the magnetic field of the plane wave, and the equivalent undulator period $\lambda_{u}$ by $\lambda_{L}$, the wave length of the plane wave, both of them being their values in the lab frame. However, we must remember that the implication is very different, the equivalent undulator period is actually about half of the wavelength of the plane wave, resulting therefore in a much shorter wavelength for the synchrotron radiation or scattered wave,

$$
\frac{\lambda_{s}}{\lambda_{L}}=\frac{\lambda_{s}}{\lambda_{u}} \frac{\lambda_{u}}{\lambda_{L}}=\frac{1+\frac{1}{4}(1+\beta) K_{u}^{2}+\frac{1}{2} \beta(1+\beta) \gamma^{2} \theta_{\ell}^{2}}{\beta(1+\beta) \gamma^{2}} \frac{\beta}{1+\beta}=\frac{1+\frac{1}{4}(1+\beta) K_{u}^{2}+\frac{1}{2} \beta(1+\beta) \gamma^{2} \theta_{\ell}^{2}}{(1+\beta)^{2} \gamma^{2}} .
$$

This is to be compared with the general result of Compton scattering in Eq. (2.12). We see that the two expressions are exactly the same to all orders of $(1-\beta)$ provided $h=0$ and $K_{u}=0$. On the one hand, it is not unexpected that we require $h=0$ in the comparison, because the above undulator theory is classical while the treatment of Compton scattering is quantum mechanical. On the other hand, it is not clear why we require the undulator parameter $K_{u}$ to be zero, since $K_{u}$ is the center of the undulator theory. Here, the undulator parameter depends the intensity of the laser beam through its magnetic field. However, the Compton scattering theory discussed above is merely kinematic together with the the quantum nature of the photon. Because of this, the frequency of the Compton scattered photon should not depend on the intensity photon beam at all. We are going to show that the equivalent undulator parameter $K$ for a high intensity optical laser beam is rather small. Then if $K$ can be neglected, the undulator theory discussed above does serve as an accurate interpretation of the inverse Compton scattering process.

The intensity of a laser beam $I_{L}$ can be defined as the energy per cross-sectional area traversed per unit time. Thus $I_{L}$ is the same as the Poynting vector,

$$
I_{L}=|\vec{E} \times \vec{H}|=\frac{1}{\mu_{0}}|\vec{E} \times \vec{B}|
$$


Since the center of the laser beam can be viewed as a plane wave, we can substitute for $E=c B$ to obtain

$$
I_{L}=\frac{c^{2} B^{2}}{Z_{0}}
$$

For a high intensity laser beam of $I_{L}=10^{19} \mathrm{~W} / \mathrm{m}^{2}, B=204.5$ Tesla. For an optical laser beam of wavelength* $\lambda_{L}=10000 \AA\left(f_{L}=3 \times 10^{14} \mathrm{~Hz}\right)$, we obtain an undulator parameter $K=0.02$. The smallness of the undulator is the result of the small wavelength of the laser beam

\section{Power of Plane Wave}

From the Maxwell's equations

$$
\vec{\nabla} \times \vec{H}=-\frac{\partial \vec{B}}{\partial t} \quad \text { and } \quad \vec{\nabla} \times \vec{E}=\vec{j}+\frac{\partial \vec{D}}{\partial t},
$$

one can easily obtain

$$
\vec{\nabla} \cdot(\vec{E} \times \vec{H})=-\frac{\partial \vec{B}}{\partial t} \cdot \vec{H}-\vec{E} \cdot \vec{j}-\vec{E} \cdot \frac{\partial \vec{D}}{\partial t}
$$

We now integrate over a volume $V$ enclosed by the area $S$ to arrive at

$$
-\int_{V} \vec{E} \cdot \vec{j} d V=\frac{\partial}{\partial t} \int_{V} \frac{1}{2}(\vec{H} \cdot \vec{B}+\vec{E} \cdot \vec{D}) d V+\int_{S}(\vec{E} \times \vec{H}) \cdot d \vec{S}
$$

where the medium over which the integration is performed is assumed to be linear. The the left side is recognized as negative rate of Joule heat losses or rate at which an electromotive force is doing work on the system. The first term on the right side represents the rate of increase of electric field and magnetic field energy inside $V$, while the second term is recognized as the rate at which field energy is going out of the volume through the area $S$. This is just an equation of energy balance and each side has the dimension of power.

For a plane wave in vacuum, we can substitute for

$$
\omega \vec{B}=\vec{k} \times \vec{E}, \quad \vec{D}=\epsilon_{0} \vec{E}, \quad \vec{B}=\mu_{0} \vec{H}
$$

\footnotetext{
*Optical wavelength ranges between 3800 and $7500 \AA$.
} 
so that the above becomes

$$
\begin{aligned}
-\int_{V} \vec{E} \cdot \vec{j} d V & =\frac{\partial}{\partial t} \int_{V} \frac{1}{2}\left(\frac{B^{2}}{\mu_{0}}+\epsilon_{0} E^{2}\right) d V+\frac{1}{\mu_{0}} \int_{S}(\vec{E} \times \vec{B}) \cdot d \vec{S} \\
& =\frac{\partial}{\partial t} \int_{V} \frac{c B^{2}}{Z_{0}} d V+\int_{S} \frac{c^{2} B^{2}}{Z_{0}} d S
\end{aligned}
$$

For a plane wave is there is no rate of increase of magnetic and electric field energy in a volume; i.e., the first term on the right side vanishes. The integral over the surface is just the power $P_{L}$ of the plane wave. The intensity of the plane wave is defined as the energy passing through a unit cross-sectional area per unit time and is therefore

$$
I_{L}=\frac{P_{L}}{S}=\frac{c^{2} B^{2}}{Z_{0}} .
$$

\section{Radiation Intensity}

Let $N_{x}$ be the number of photon radiated. The rate at which the radiation take place is

$$
\frac{d N_{x}}{d t}=f \frac{N_{e} N_{L}}{4 \pi \sigma_{x} \sigma_{z}} \sigma_{T}
$$

where $N_{e}$ and $N_{L}$ are, respectively, the numbers of electrons and photon in the electron bunch and laser pulse, $\sigma_{x, y}$ are the rms transverse beam sizes, $f$ is the frequency of collision, and

$$
\sigma_{T}=\frac{8 \pi}{3} r_{e}^{2}
$$

is the total Thomson cross section and $r_{e}=e^{2} /\left(4 \pi \epsilon_{0} m c^{2}\right)$ is the electron classical radius. Note that $4 \pi \sigma_{x} \sigma_{y}$ is the effective cross section of the electron beam. We have taken $2 \sigma_{x}$ and $2 \sigma_{y}$ as the radii of the elliptical area. We wish to point out that whether we take $\pi \sigma_{x} \sigma_{y}$ or $4 \pi \sigma_{x} \sigma_{y}$ as the effective cross-section of the laser pulse does not affect the final result that we are going to derive. What we use later in Eq. (6.8) is to relate the intensity of the laser pulse (power per unit cross-sectional area) to the magnetic field strength $B$ of the pulse.

We are interested in the number of photons radiated, $N_{x}$, per electron during one collision passage into a solid angle. For this we need the differential Thomas cross-section per unit solid angle $\Omega_{e}$ in the electron rest frame, or

$$
\frac{d \sigma}{d \Omega_{e}}=\frac{r_{e}^{2}}{2}\left(1+\cos ^{2} \theta_{e}\right),
$$


which obviously integrate to the total Thomas cross-section. Note that the angular distribution here is given in the traditional frame of Thomas scattering, or the frame where the electron is initially at rest. The number of photon emitted in a solid angle per electron per collision is therefore

$$
\frac{d N_{x}}{d \Omega_{e}}=\frac{1}{f N_{e}} \frac{d N_{x}}{d t}=\frac{N_{L}}{4 \pi \sigma_{x} \sigma_{y}} \frac{r_{e}^{2}}{2}\left(1+\cos ^{2} \theta_{e}\right) .
$$

Now the energy in the laser pulse of duration $\tau$ is $\mathcal{E}_{L}=N_{L} h f_{L}$, which is equal to $P_{L} \tau_{L}$, where $P_{L}$ is the power of the laser beam. In terms of these new variables,

$$
\frac{d N_{x}}{d \Omega_{e}}=\frac{P_{L} \tau_{L}}{4 \pi \sigma_{x} \sigma_{y} h f_{L}} \frac{r_{e}}{2} \frac{e^{2}}{4 \pi \epsilon_{0} m c^{2}}\left(1+\cos ^{2} \theta_{e}\right)=\frac{P_{L} \tau_{L}}{4 \pi \sigma_{x} \sigma_{y} f_{L}} \frac{\alpha r_{e}}{4 \pi m c}\left(1+\cos ^{2} \theta_{e}\right),
$$

where

$$
\alpha=\frac{e^{2}}{4 \pi \epsilon_{0} \hbar c}=\frac{e^{2}}{2 \epsilon_{0} h c} \approx \frac{1}{137}
$$

is the fine-structure constant. The effective number of undulator periods is defined as

$$
N_{\mathrm{eff}}=\frac{c \tau_{L}}{\lambda_{L}}=f_{L} \tau_{L}
$$

The strength of the magnetic component $B$ in the laser beam is related to the power of the beam by

$$
\frac{P_{L}}{4 \pi \sigma_{x} \sigma_{y}}=\frac{c B^{2}}{\mu_{0}}
$$

Then

$$
\frac{d N_{x}}{d \Omega_{e}}=\frac{N_{\mathrm{eff}} \lambda_{L}^{2}}{c} \frac{c B^{2}}{\mu_{0}} \frac{\alpha}{4 \pi m c^{2}} \frac{e^{2}}{4 \pi \epsilon_{0} m c^{2}}\left(1+\cos ^{2} \theta_{e}\right)=\frac{\alpha N_{\mathrm{eff}} K_{u}^{2}}{4}\left(1+\cos ^{2} \theta_{e}\right),
$$

where the undulator parameter

$$
K_{u}=\frac{e B \lambda_{L}}{2 \pi m c}
$$

has been used. Integrating over all outgoing directions, the total number of photons produced is

$$
N_{x}=\frac{4 \pi}{3} \alpha N_{\mathrm{eff}} K_{u}^{2}
$$

To compare with an undulator theory, we need to transform the above to the angular distribution in the lab frame. The transformation is from the solid angle $d \Omega_{e}=\sin \theta_{e} d \theta_{e} d \varphi_{e}$ to $d \Omega_{\ell}=\sin \theta_{\ell} d \theta_{\ell} d \varphi_{\ell}$, where we have denoted the polar angle in the lab frame as $\theta_{\ell}$ and is connected to $\theta_{e}$ by Eq. (2.7). Note that although the azimuthal angle $\varphi$ does not change in 
a transformation along the polar axis, however, the polar axis does reverse direction in these two frames. Thus $\varphi_{e}=2 \pi-\varphi_{\ell}$ or $d \varphi_{e}=-d \varphi_{\ell}$. We have

$$
\frac{d \cos \theta_{e}}{d \theta_{\ell}}=\frac{\sec \theta_{\ell} \tan \theta_{\ell}\left[2 \gamma^{2} \beta \sec \theta_{\ell}+\gamma^{2} \sec ^{2} \theta_{\ell}+\gamma^{2}-1\right]}{\left(1+\gamma^{2} \tan ^{2} \theta_{\ell}\right)^{2}}
$$

or

$$
\frac{d \Omega_{e}}{d \Omega_{\ell}}=-\frac{d \cos \theta_{e}}{d \cos \theta_{\ell}}=\frac{\sec ^{2} \theta_{\ell}\left[2 \gamma^{2} \beta \sec \theta_{\ell}+\gamma^{2} \sec ^{2} \theta_{\ell}+\gamma^{2}-1\right]}{\left(1+\gamma^{2} \tan ^{2} \theta_{\ell}\right)^{2}}
$$

Because of the $\left(1+\gamma^{2} \tan ^{2} \theta_{\ell}\right)^{2}$ in the denominator, the polar angle $\theta_{\ell}$ in the lab frame is restricted to $\sim \gamma^{-1}$, when $\gamma \gg 1$. How about the angle $\pi-\theta_{\ell}$, the scattered angle in the direction of the laser beam? The expression inside the squared-brackets in the numerator gives [ ] $\rightarrow-1 /\left(4 \gamma^{2}\right)$ at $\theta_{\ell}=\pi$, whereas the same factor gives [ ] $\rightarrow 4 \gamma^{2}$ when $\theta_{\ell}=0$. The implication is that all the scattered photon are concentrated in the reverse laser direction within a cone of opening angle $\gamma^{-1}$, but not in the reverse direction. This is just a kinematic result of Lorentz transformation. Thus the transformation of differential solid angles can be rewritten as

$$
\frac{d \Omega_{e}}{d \Omega_{\ell}}=\frac{4 \gamma^{2}\left(1+\frac{3}{2} \theta_{\ell}^{2}\right)}{\left(1+\gamma^{2} \theta_{\ell}^{2}\right)^{2}} \rightarrow \frac{4 \gamma^{2}}{\left(1+\gamma^{2} \theta_{\ell}^{2}\right)^{2}},
$$

where the term $\frac{3}{2} \theta_{\ell}^{2}$ is neglected because it contributes only $\mathcal{O}\left(\gamma^{-2}\right)$. Thus the differential scattered photon intensity in the lab frame becomes

$$
\frac{d N_{x}}{d \Omega_{\ell}}=\frac{2 \alpha N_{\mathrm{eff}} K_{u}^{2} \gamma^{2}}{\left(1+\gamma^{2} \theta_{\ell}^{2}\right)^{2}}
$$

Notice that the integration of the above expression over the solid angle $\Omega_{\ell}\left(\theta_{\ell}\right.$ from 0 to $\pi$ and $\varphi_{\ell}$ from 0 to $2 \pi$ ) does not reproduce $N_{x}$ in Eq. (6.11). This is a not real surprise because the above expression [Eq. (6.15)] has been simplified. If we wish to obtain the correct result of Eq. (6.11), we need to let $\sin \theta_{\ell} d \theta_{\ell} \approx \frac{1}{2} d \theta_{\ell}^{2}$ and integrate $\theta_{\ell}=0$ to

$$
\theta_{1}=\frac{\sqrt{e^{2 / 3}-1}}{\gamma}=\frac{0.974}{\gamma}
$$

which demonstrates that the opening angle is $\sim \gamma^{-1}$. Another method that is usually made is to assume the differential intensity to be constant at the exact forward direction of the electron (i.e., $\theta_{\ell}=0$ ) within a small opening solid angle, and multiply it by the small opening solid angle to obtain the total number of photons emitted. To match the result of Eq. (6.11), this small opening solid angle is $\Delta \Omega_{\ell}=\pi \theta_{2}^{2}=\pi /\left(3 \gamma^{2}\right)$, or the opening angle in each transverse direction is $\theta_{2}=1 /(\sqrt{3} \gamma)$. 


\section{Differential Undulator Emission Intensity}

In this section, we compute the intensity of the emitted per electron while passing through an undulator, starting from the theory of synchrotron radiation. The differential intensity observed at a point $P$ is

$$
\frac{d^{2} N}{d \Omega_{\ell} d \omega}=\frac{r_{e} m c \omega^{2}}{4 \pi}\left|\int_{-\infty}^{\infty} \vec{n} \times(\vec{n} \times \vec{\beta}) e^{-i \omega\left(t_{r}-\vec{n} \cdot \vec{r}_{p} / c\right)} d t_{r}\right|^{2},
$$

for an electron moving with velocity $\vec{\beta} c$, where $r_{e}$ is the classical electron radius. Here $\vec{r}_{p}$ is the vector from the origin of the reference frame to electron and $\vec{n}$ is the unit vector pointing from the electron to the point of observation $P$. The frequency $\omega$ varies from 0 to $\infty$ (negative frequency has been included already). All quantities are to be taken at the retarded time $t_{r}$ at the electron. The origin of the reference frame is located at the center of the undulator, while the length of the undulator is running in the $z$-direction, which is the direction of the electron before entering into the undulator.

We consider the undulator to be helical in order to simplify the derivation. Let us introduce the unit vectors

$$
\hat{e}_{ \pm}=\frac{\hat{x} \pm i \hat{y}}{\sqrt{2}}
$$

which satisfies the properties

$$
\hat{e}_{ \pm}=\hat{e}_{\mp}^{*}, \quad \hat{e}_{ \pm} \cdot \hat{e}_{ \pm}=0, \quad \hat{e}_{ \pm} \cdot \hat{e}_{\mp}=\hat{e}_{ \pm} \cdot \hat{e}_{ \pm}^{*}=1
$$

The spiraling magnetic field $\vec{B}_{u}$ of the undulator can be generated from the vector potential

$$
\vec{A}_{u}=\frac{A_{u}}{\sqrt{2}}\left[\hat{e}_{-} e^{i k_{u} z}+\text { c.c. }\right]=A_{u}\left[\hat{x} \cos k_{u} z+\hat{y} \sin k_{u} z\right]
$$

where $\lambda_{u}=1 / k_{u}$ is the period of the undulator. Thus

$$
\vec{B}_{u}=\vec{\nabla} \times \vec{A}_{u}=-\frac{k_{u} A_{u}}{\sqrt{2}}\left[\hat{e}_{-} e^{i k_{u} z}+\text { c.c. }\right]
$$

where $B_{u}=-k_{u} A_{u}$ is the strength of the spiraling undulator magnetic field. The canonical momentum $\vec{p}=\gamma m \vec{v}-e \overrightarrow{A_{u}}$ is conserved. Before entering the undulator, $p_{\perp}=0$. Thus inside the undulator

$$
\vec{\beta}_{\perp}=\frac{e \vec{A}_{u}}{\gamma m c}=\frac{e A_{u}}{\gamma m c} \frac{1}{\sqrt{2}}\left[\hat{e}_{-} e^{i k_{u} z}+\text { c.c. }\right]=-\frac{K_{u}}{\gamma} \frac{1}{\sqrt{2}}\left[\hat{e}_{-} e^{i k_{u} z}+\text { c.c. }\right]
$$


where

$$
K_{u}=-\frac{e A_{u}}{\gamma m c}=\frac{e B_{u}}{\gamma m c k_{u}}
$$

is the undulator parameter. Note that unlike the planar undulator, here $\beta_{\perp}=\left|\vec{\beta}_{\perp}\right|$ is a constant inside the helical undulator. The longitudinal velocity of the electron inside the undulator is given by

$$
\beta_{\|}^{2}=\beta^{2}-\beta_{\perp}^{2}=\beta^{2}-\frac{K_{u}^{2}}{\gamma^{2}}=1-\frac{1+K_{u}^{2}}{\gamma^{2}}
$$

and is also a constant inside the helical undulator. We obtain the longitudinal position $z\left(t_{r}\right)=\beta_{\|} t_{r}$. Substitution into Eq. (7.22) and integration gives the position vector $\vec{r}_{p}$ of the electron

$$
\vec{r}_{p}=\frac{i K_{u} c}{\sqrt{2} \gamma \omega_{u}}\left[\hat{e}_{-} e^{i \omega_{u} t_{r}}-\text { c.c. }\right]+\hat{z} \beta_{\|} c t_{r},
$$

where $\omega_{u}=k_{u} \beta_{\|} c$ is the angular frequency of the undulator. The constant of integration has been chosen in such a way that the origin of the reference frame is right at the center of the undulator, which spans from $z=-\frac{1}{2} N_{u} \lambda_{u}$ to $+\frac{1}{2} N_{u} \lambda_{u}$, where $N_{u}$ is the total number of undulator periods and $\lambda_{u}$ is the undulator period.

The point of observation $P$ is assumed to be very far away from the undulator. It is represented by the vector $\vec{R}$. Since the width and length of the undulator is very much smaller than $R$, we make the assumption that the unit vector $\hat{n}$ from the electron to $P$ is always in the direction of $\vec{R}$, or $\vec{R}=\hat{n} R$. Thus the polar angle $\theta_{\ell}$ and azimuthal angle $\varphi_{\ell}$ at the point $P$ are assumed to be constant. In other words, the unit vector

$$
\hat{n}=\hat{x} \sin \theta_{\ell} \cos \varphi_{\ell}+\hat{y} \sin \theta_{\ell} \sin \varphi_{\ell}+\hat{z} \cos \theta_{\ell}=\frac{\sin \theta_{\ell}}{\sqrt{2}}\left[\hat{e}_{-} e^{i \varphi_{\ell}}+\text { c.c. }\right]+\hat{z} \cos \theta_{\ell},
$$

is assumed to be time independent. We are now in the position of computing the synchrotron radiation using Eq. (7.17). First, we compute

$$
\hat{n} \times(\hat{n} \times \vec{\beta})=\hat{n}(\hat{n} \cdot \vec{\beta})-\vec{\beta}
$$

With

$$
\begin{aligned}
\hat{n} \cdot \vec{\beta} & =-\frac{K_{u}}{2 \gamma} \sin \theta_{\ell}\left[\hat{e}_{-} e^{i \varphi_{\ell}}+\text { c.c. }\right] \cdot\left[\hat{e}_{-} e^{i \omega_{u} t_{r}}+\text { c.c. }\right]+\beta_{\|} \cos \theta_{\ell} \\
& =-\frac{K_{u}}{\gamma} \sin \theta_{\ell} \cos \left(\omega_{u} t_{r}-\varphi_{\ell}\right)+\beta_{\|} \cos \theta_{\ell},
\end{aligned}
$$


we obtain

$$
\begin{aligned}
\hat{n} \times(\hat{n} \times \vec{\beta})= & \frac{1}{\sqrt{2}}\left[\hat{e}_{-} e^{i \varphi_{\ell}}+\text { c.c. }\right]\left[-\frac{K_{u}}{\gamma} \sin ^{2} \theta_{\ell} \cos \left(\omega_{u} t_{r}-\varphi_{\ell}\right)+\frac{\beta_{\|}}{2} \sin 2 \theta_{\ell}\right] \\
& +\frac{K_{u}}{\sqrt{2} \gamma}\left[\hat{e}_{-} e^{i \omega_{u} t_{r}}+\text { c.c. }\right]-\hat{z}\left[\sin ^{2} \theta_{\ell}+\frac{K_{u}}{2 \gamma} \sin 2 \theta_{\ell} \cos \left(\omega_{u} t_{r}-\varphi_{\ell}\right)\right] .
\end{aligned}
$$

We know that the synchrotron radiation will be in the forward direction of the electron concentrated within the opening angle $\theta_{\ell} \sim \gamma^{-1}$. The above messy expression can therefore be very much simplified by keeping only the first order in $\theta_{\ell}$, or

$$
\hat{n} \times(\hat{n} \times \vec{\beta})=\frac{1}{\sqrt{2}}\left[\hat{e}_{-}\left(\beta_{\|} \theta_{\ell} e^{i \varphi_{\ell}}+\frac{K_{u}}{\gamma} e^{i \omega_{u} t_{r}}\right)+\text { c.c. }\right]-\hat{z} \frac{K_{u} \theta_{\ell}}{\gamma} \cos \left(\omega_{u} t_{r}-\varphi_{\ell}\right) .
$$

The exponent in the synchrotron-radiation formula is

$$
-i \omega\left(t_{r}-\frac{\hat{n} \cdot \vec{r}_{p}}{c}\right)=i \frac{K_{u} \omega}{\gamma \omega_{u}} \sin \theta_{\ell} \cos \left(\omega_{u} t_{r}-\varphi_{\ell}\right)-i \omega t_{r}\left(1-\beta_{\|} \cos \theta_{\ell}\right)
$$

This can be readily simplified using the small-opening-angle approximation. Notice that

$$
1-\beta_{\|} \cos \theta_{\ell} \approx 1-\sqrt{1-\frac{1+K_{u}^{2}}{\gamma^{2}}}\left(1-\frac{\theta_{\ell}^{2}}{2}\right) \approx \frac{1+K_{u}^{2}+\gamma^{2} \theta_{\ell}^{2}}{2 \gamma^{2}}=\frac{\omega_{u}}{\omega_{1}},
$$

where $\omega_{1}$ denotes the fundamental emission frequency from the helical undulator. The corresponding wavelength is $\lambda_{1}=2 \pi c / \omega_{1}$, which is to be compared with $\lambda_{s}$ in Eq. (4.5) in a planar undulator. There we have $K_{u}^{2} / 2$ in the numerator instead of just $K_{u}^{2}$ here in Eq. (7.32). The exponent then simplifies to

$$
-i \omega\left(t_{r}-\frac{\hat{n} \cdot \vec{r}_{p}}{c}\right)=i \frac{\omega}{\omega_{u}} \frac{K_{u} \theta_{\ell}}{\gamma} \cos \left(\omega_{u} t_{r}-\varphi_{\ell}\right)-i \frac{\omega_{u}}{\omega_{1}} \omega t_{r}
$$

We are now ready to perform the integration over $t_{r}$ in the differential intensity formula of Eq. (7.17). It is clear that the term containing $\cos \left(\omega_{u} t_{r}-\varphi_{\ell}\right)$ in the exponent [Eq. (7.33)] will lead to a summation over Bessel functions and higher harmonics for the undulator emission, and as a result complicated mathematical expressions. Fortunately, all these can be avoided by setting $\theta_{\ell}=0$, or to restrict ourselves to the computation of the differential intensity of the synchrotron emission in the exact forward direction of the incident electron. We then obtain the simplified expression

$$
\begin{aligned}
{\left[\hat{n} \times(\hat{n} \times \vec{\beta}) e^{-i \omega\left(t_{r}-\vec{n} \cdot \vec{r}_{p} / c\right)}\right]_{\theta_{\ell}=0} } & =\frac{K_{u}}{\sqrt{2} \gamma}\left[\hat{e}_{-} e^{i \omega_{u} t_{r}}+\text { c.c. }\right] e^{-i\left(\omega_{u} \omega / \omega_{1}\right) t_{r}} \\
& =\frac{K_{u}}{\sqrt{2} \gamma}\left[\hat{e}_{-} e^{-i\left(\omega / \omega_{1}-1\right) \omega_{u} t_{r}}+\hat{e}_{+} e^{-i\left(\omega / \omega_{1}+1\right) \omega_{u} t_{r}}\right] .
\end{aligned}
$$


The integration over $t_{r}$ is now attempted over the range of the undulator from $t_{r}=-\pi N_{u} / \omega_{u}$ to $+\pi N_{u} / \omega_{u}$ (remembering that the origin of the coordinate system has been placed at the center of the undulator), where $N_{u}$ is the number of undulator periods and $2 \pi / \omega_{u}$ is the undulator wavelength or undulator period in time. The integration readily gives

$$
\begin{aligned}
\int_{-\pi N_{u} / \omega_{u}}^{\pi N_{u} / \omega_{u}}\left[\hat{n} \times(\hat{n} \times \vec{\beta}) e^{-i \omega\left(t_{r}-\vec{n} \cdot \vec{r}_{p} / c\right)}\right]_{\theta_{\ell}=0} d t_{r} \\
=\int_{-\pi N_{u}}^{\pi N_{u}} \frac{K_{u}}{\sqrt{2} \gamma \omega_{u}}\left[\hat{e}_{-} e^{-i\left(\omega / \omega_{1}-1\right) \omega_{u} t_{r}}+\hat{e}_{+} e^{-i\left(\omega / \omega_{1}+1\right) \omega_{u} t_{r}}\right] d\left(\omega_{u} t_{r}\right) \\
=\frac{\sqrt{2} \pi K_{u} N_{u}}{\gamma \omega_{u}}\left[\hat{e}_{-} \frac{\sin \pi N_{u}\left(\omega / \omega_{1}+1\right)}{\pi N_{u}\left(\omega / \omega_{1}+1\right)}+\hat{e}_{+} \frac{\sin \pi N_{u}\left(\omega / \omega_{1}-1\right)}{\pi N_{u}\left(\omega / \omega_{1}-1\right)}\right]
\end{aligned}
$$

This is finally substituted into the differential intensity formula of Eq. (7.17) to obtain the number of radiated photons emitted per electron per unit angular frequency and per unit solid angle

$$
\begin{aligned}
\left.\frac{d N_{x}}{d \omega d \Omega_{\ell}}\right|_{\theta_{\ell}=0} & =\left.\frac{1}{\hbar \omega_{1}} \frac{d W}{d \omega d \Omega_{\ell}}\right|_{\theta_{\ell}=0}=\frac{\alpha \omega^{2}}{4 \pi^{2} \omega_{1}}\left|\int \hat{n} \times(\hat{n} \times \vec{\beta}) e^{-i \omega\left(t_{r}-\vec{n} \cdot \vec{r}_{p} / c\right)} d t_{r}\right|_{\theta_{\ell}=0}^{2} \\
& =\frac{\alpha \omega^{2} K_{u}^{2} N_{u}^{2}}{2 \gamma^{2} \omega_{u}^{2} \omega_{1}}\left[\left|\frac{\sin \pi N_{u}\left(\omega / \omega_{1}+1\right)}{\pi N_{u}\left(\omega / \omega_{1}+1\right)}\right|^{2}+\left|\frac{\sin \pi N_{u}\left(\omega / \omega_{1}-1\right)}{\pi N_{u}\left(\omega / \omega_{1}-1\right)}\right|^{2}\right],
\end{aligned}
$$

where we have made substitutions for the classical electron radius and the fine-structure constant

$$
r_{e}=\frac{e^{2}}{4 \pi \epsilon_{0} m c^{2}}, \quad \alpha=\frac{e^{2}}{4 \pi \epsilon_{0} \hbar c}, \quad \text { or } \quad \frac{r_{e}}{\alpha}=\frac{\hbar}{m c} .
$$

The sinc functions in Eq. (7.35) indicate that the variation of the emission frequency $\omega$ is limited to within

$$
\left|\frac{\omega}{\omega_{1}} \pm 1\right| \lesssim \frac{1}{N_{u}}
$$

where the positive/negative sign is for the first/second sinc function. Since the number of undulator periods $N_{u}$ is usually is a rather larger number, the sinc functions are therefore approximately $\delta$-functions; i.e.,

$$
\int_{0}^{\infty}\left|\frac{\sin \pi N_{u}\left(\omega / \omega_{1} \pm 1\right)}{\pi N_{u}\left(\omega / \omega_{1} \pm 1\right)}\right|^{2} \frac{d \omega}{\omega_{1}} \approx \frac{1}{N_{u}} \int_{0}^{\infty} \delta\left(\frac{\omega}{\omega_{1}} \pm 1\right) \frac{d \omega}{\omega_{1}}=\left\{\begin{array}{cc}
0 & +\operatorname{sign} \\
\frac{1}{N_{u}} & -\operatorname{sign}
\end{array}\right.
$$

The differential intensity of synchrotron radiation can therefore be written as

$$
\left.\frac{d N_{x}}{d \omega d \Omega_{\ell}}\right|_{\theta_{\ell}=0}=\frac{\alpha \omega^{2} K_{u}^{2} N_{u}^{2}}{2 \gamma^{2} \omega_{u}^{2} \omega_{1}}\left[\frac{\sin \pi N_{u}\left(\omega / \omega_{1}-1\right)}{\pi N_{u}\left(\omega / \omega_{1}-1\right)}\right]^{2}
$$


and

$$
\left.\frac{d N_{x}}{d \Omega_{\ell}}\right|_{\theta_{\ell}=0}=\frac{\alpha \omega_{1}^{2} K_{u}^{2} N_{u}}{2 \gamma^{2} \omega_{u}^{2}}=\frac{2 \alpha K_{u}^{2} N_{u} \gamma^{2}}{\left(1+K_{u}^{2}\right)^{2}}
$$

when the integration over $\omega$ is performed. The last expression is to be compared with the differential scattered intensity of Thomson scattering in Eq. (6.15). The difference is the extra factor of $\left(1+K_{u}^{2}\right)^{2}$ in the denominator of Eq. (7.41). This factor comes about because the different definitions of $\omega_{1} / \omega_{u}$ in the two theories:

$$
\frac{\omega_{1}}{\omega_{u}}=\left\{\begin{array}{cl}
2 \gamma^{2} & \text { Thomson scattering, } \\
\frac{2 \gamma^{2}}{1+K_{u}^{2}} & \text { undulator theory. }
\end{array}\right.
$$

To obtain the total number of photons emitted, we need to integrate over the solid angle $\Omega_{\ell}$. Since we have here only the differential intensity at $\theta_{\ell}=0$, it is unsure about the small solid angle that we need to multiply Eq. (7.41) with. One may follow the experience from Thomson scattering and multiply it by the small solid angle $\Delta \Omega_{\ell}=\pi /\left(3 \gamma^{2}\right)$. However, this need not be correct because although the angular distributions for the two theories are roughly the same when $\theta_{\ell} \lesssim \gamma^{-1}$, they may not exactly the same at larger opening angles.

\section{Appendix}

Transforming from the frame where the electron is initially at rest to the lab frame, the momentum of the scattered photon has a longitudinal component and transverse component equal to

$$
\begin{aligned}
\left(\frac{h f_{s}}{c}\right)_{\|} & =\gamma\left(-\cos \theta_{e}+\beta\right) \frac{h f_{s 0}}{c} . \\
\left(\frac{h f_{s}}{c}\right)_{\perp} & =\sin \theta_{e} \frac{h f_{s 0}}{c} .
\end{aligned}
$$

Thus the scattered angle (in the direction of electron) in the lab frame is

$$
\tan \theta_{\ell}=\frac{\sin \theta_{e}}{\gamma\left(-\cos \theta_{e}+\beta\right)} .
$$

Writing $\sin \theta_{e}=\sqrt{1-\cos ^{2} \theta_{e}}$ and solving the quadratic equation results in Eq. (2.7). 


\section{References}

[1] H. Wiedermann, Synchrotron Radiation, SLAC-R-637, 2000.

[2] Y. Hua, Lecture Notes. 